Editorial

\title{
Editorial to the Inaugural Edition of the Journal of Legal Research Methodology on 'Virtual Research Methodology'
}

\author{
Paul Dargue, Anqi Shen, Lyndsey Bengtsson, Andrew Watson
}

We launched the Journal of Legal Research Methodology with the aim of providing a forum for discussion and delineation of different methodological approaches to the study of law. To date, we felt, there has been an alarming lack of 'self-conscious reflection' upon the methods we employ when conducting all kinds of legal research. Whenever a claim is made that some form of knowledge is being generated or created, which happens in almost all legal research, and certainly all 'empirical' research which produces data, it must be asked how that data - information, knowledge or insight were generated. Authors, we think, have a duty to reflect closely upon the methods they use and what decisions they made and what drove them. Doing so will validate and also strengthen the results if the methods have been sufficiently critiqued.

What does critiquing one's methods mean? In part it means building ironmen, not strawmen. That is, when reaching conclusions the researcher must confront the strongest arguments against their theory (rather than the weakest), and must explore the limitations in their approach and explain how, despite these, their approach is still valid, the findings are reliable, may have wider application and / or contributes to scholarship. It also means accurately and fully, as far as possible, describing the research process and explaining what issues were encountered, rather than only the positive things, and how that altered the approach. Authors should, where appropriate, consider matters around reliability, replicability, validity, and objectivity, and explain how these have been met, or why they do not apply.

The Covid-19 has changed legal research over the last eighteen months. Research conferences ground to a halt, face-to-face collaboration ceased, research needed to be conducted from home, often under lockdown. We invited submissions to the inaugural edition of this Journal exploring how this situation has impacted legal 
research. The result of our call is the four wonderful contributions presented in this edition.

The edition begins with Allsopp et al's 'Observing Data-Driven Approaches to Covid19: Reflections from a Distributed, Remote, Interdisciplinary, Research Project'. The authors of this paper provide insightful reflections on how the pandemic impacted their distinct roles in the AHRC funded research project exploring data-driven approaches to Covid-19. The article provides valuable insight, and advice, on conducting crossdisciplinary, cross-institutional, research on Covid.

Klinker and Smith's article, 'From Law to Policy and Practice - Collaborative Research Amidst the Pandemic: The Creation of the Bournemouth Protocol on Mass Grave Protection and Investigation' shares fascinating reflections on a project interrupted by the pandemic. The article recounts the trials of building collaborations across borders, disciplines and objectives, all during a pandemic.

Unnithan's article 'Dialling in: Reflections on Telephone Interviews in light of the Covid19 Pandemic' will be beneficial for those considering alternative methods of the faceto-face interview. The under-explored topics of telephone interviews is given reflective critique here, offering very useful sources of information for later researchers employing this method.

Bancroft's article 'Domestic Violence Legislation, Virtual Legal Methods and Researching One Female Teacher's Lived Experiences of Recovery from Intimate Partner Violence During the COVID-19 Global Pandemic' provides her critical reflections upon conducting a zoom-interview from home during the pandemic. The article makes particularly important contributions to the discussion around ensuring ethical practices when conducting interviews into sensitive topics.

These well-argued articles strongly demonstrate the determination, diligence and resilience of legal researchers who strive, in these challenging times, to make contributions to the disciplines in law, the academic communities and the legal world.

We thank all the authors, reviewers, and library staff for the terrific response we received to the call for papers and look forward to publishing many more editions of the Journal. 